$\mathrm{ms}^{2}$ vs $\left.885 \pm 523 \mathrm{~ms}^{2} ; \mathrm{p}<0.02\right)$, HF $\left(252 \pm 280 \mathrm{~ms}^{2}\right.$ vs $560 \pm 657$ $\left.\mathrm{ms}^{2} ; \mathrm{p}<0.005\right)$ and a tendency of difference in LF/HF $(2.5 \pm 0.7$ vs $1.9 \pm 1$; ns). These data are consistent with reduced vagal function in RA patients. In RA, IL-1 $\beta$ levels in CSF were markedly higher than serum levels $(8.35 \pm 12.59$ vs $0.00 \pm 0.06 \mathrm{pg} /$ $\mathrm{ml} ; \mathrm{p}<0.005)$ indicating intrathecal production. In contrast, CSF IL-6 levels were lower in CSF than serum $(2.59 \pm 1.43 \mathrm{pg} /$ $\mathrm{ml}$ vs $6.25 \pm 4.21 \mathrm{pg} / \mathrm{ml}, \mathrm{p}=0.01)$. CSF IL-1 $\beta$, but not IL- 6 , correlated positively with HRV, as measured with $\mathrm{LF} / \mathrm{HF}$ ( $\mathrm{r}=0.64$; $\mathrm{p}<0.05)$. Serum IL-1 $\beta$ was not correlated to HRV, but serum IL- 6 correlated inversely to LF both in RA $(r=-0,55 \mathrm{p}<0.05)$ and controls $(\mathrm{r}=-0.46 ; \mathrm{p}<0.05)$.

Conclusions The decreased autonomic function in RA, reflected in low vagus activity and thereby reduced control of systemic inflammation, may further impair the disease. Our data indicate that intrathecal IL-1beta and possibly also other inflammatory mechanisms in the CNS may have importance for the reduced vagal function of RA. These mechanisms may constitute future targets for improvement of cholinergic immune-suppression of the disease.

\section{A PROPOSED MECHANISM FOR AUTONOMIC DYSFUNCTION IN RHEUMATOID ARTHRITIS - REDUCED VAGAL ACTIVITY RELATED TO HIGH INTRATHECAL IL-1 $\beta$ LEVELS}

Reem Altawil, ${ }^{1}$ Diana Kadetoff,, ${ }^{2}$ Marie Westman, ${ }^{1}$ Mats Jensen-Urstad, ${ }^{3}$ Magnus Andersson, ${ }^{4}$ Eva Kosek, ${ }^{2}$ Jon Lampa' ${ }^{1}$ Department of Medicine, Rheumatology Unit, Karolinska University Hospital, Karolinska Institute, Stockholm, Sweden; 2Department of Clinical Neuroscience, MR Center, Karolinska University Hospital, Karolinska Institute, Stockholm, Sweden, Karolinska University Hospital, Karolinska Institute, Stockholm, Sweden; ' ${ }^{3}$ epartment of Medicine, Cardiology unit, Karolinska University Hospital, Karolinska Institute, Stockholm, Sweden; ${ }^{4}$ Department of Clinical Neuroscience, Neuroimmunology Unit, Karolinska University Hospital, Karolinska Institute, Stockholm, Sweden

\subsection{6/annrheumdis-2011-201230.15}

Objective Rheumatoid arthritis (RA) is known to be associated with autonomic dysfunction, with a decreased vagus tone. However, the mechanisms have not been completely elucidated. Nervus vagus function is regulated by action of muscarinic receptors in the CNS (Pavlov, PNAS, 2006), and earlier investigations have shown that the pro-inflammatory cytokine interleukin (IL)-1beta may cause dysfunction in cholinergic neurotransmission (Schliebs, Brain Res 2006). Here the authors investigated autonomic activity in RA and controls in relation to serum and cerebrospinal fluid (CSF, only RA) levels of IL-1 $\beta$ and IL- 6 .

Methods Fourteen female patients with RA and 15 female age matched healthy controls (HC) were enrolled in this study. Median DAS28 score for RA patients was 3.6 (range 1.5 to 6.6). No patient/control had any neurological or heart disorder. Heart rate variability (HRV) was measured with Holter ECG over 24 $h$ using spectral analysis of Low frequency (LF) power (0.04 to $0.15 \mathrm{~Hz})$, High frequency power (HF) $(0.15$ to $0.40 \mathrm{~Hz})$ and the ratio of $\mathrm{LF}$ and $\mathrm{HF}(\mathrm{LF} / \mathrm{HF})$. The measures were calculated from an entire $24 \mathrm{~h}$ ambulatory ECG recording. Lumbar puncture was performed in RA patients only and CSF was analysed with high sensitivity ELISA for IL-1 $\beta$ and IL-6. Serum samples were analysed with ELISA for IL-1 $\beta$ and IL- 6 .

Results There were significant differences between RA and $\mathrm{HC}$ in heart rate $(74 \pm 6$ vs $66 \pm 5 \mathrm{bpm} ; \mathrm{p}<0.001)$, LF $(522 \pm 213$ 